\title{
EDITORIAL
}

\section{Chance for change?}

With the number of drug approvals plummeting, and fears that current drug safety issues are slowing the regulatory process, a fundamental reassessment of how drugs are brought to the market seems crucial. Given the ever-growing time and cost of clinical development, this area looks to be particularly ripe for considering novel approaches.

As 2005 drew to a close, the tally for the widely used benchmark for industry success - the number of new molecular entities (NMEs) that have been approved by the US FDA - stood at a woeful 14 at the time of going to press. After the promising recovery from the record low of 17 NMEs in 2002 to 31 NMEs in 2004, laments about weak pipelines will again be heard far and wide.

But unlike previous years, such laments will probably be muffled by arguably more pressing concerns for the industry. Drug safety has become a huge issue in the wake of Vioxx, as sharply highlighted by the fact that the number of drugs given 'black-box' warnings by the FDA has doubled in the past year. Indeed, industry commentators have suggested that an increase in the risk aversiveness of regulatory agencies, potentially leading to requests for larger trials and more safety data, could be contributing to the downturn in new drug approvals.

The FDA accelerated approval system, a mechanism that enables promising drugs for serious and life-threatening diseases to be marketed before their clinical efficacy is firmly established, has also come under fire. This issue has been highlighted in particular in the past year by the failure of the cancer drug gefitinib (Iressa) to demonstrate a survival benefit in Phase III trials following its accelerated approval for treating patients with advanced lung cancer on the basis of surrogate endpoints. But as previously highlighted ${ }^{1}$, if early decisions on the value of investigational drugs are to be permitted to get them to patients sooner, there will be an inevitable degree of risk of making the wrong decision. In the case of diseases such as cancer, such a tradeoff seems worthwhile.

The ideal, of course, would be to find ways of improving the chances of getting valuable drugs to patients as soon as possible, while preserving safety standards. Strategies that could reduce the size of clinical trials and consequent cost are also sorely needed. In this context, one approach that is attracting increasing attention, as discussed in a review on page 27, is the use of Bayesian statistical methods in clinical research, which to many represent a radical departure from the traditional 'frequentist' approaches that have been dominant for decades.

Frequentist approaches have the virtues of rigour and narrow focus on the experimental question being asked, but their inflexibility can lead to a tendency for trials to be overly large. Furthermore, existing information - for example, from previous trials - that could be relevant to the current trial is typically overlooked because the standard approaches used cannot incorporate it. Bayesian strategies, however, allow the incorporation of prior knowledge into trials, and also for accumulating results to be assessed at any time, with the possibility of modifying the design of the trial to more efficiently answer the experimental question at hand. But although common in other fields, such as ecology, so far their uptake in clinical research is still relatively limited.

Clearly, a major barrier to further uptake could be concern that adoption of such approaches, particularly if not done rigorously, increases the risk of making the wrong regulatory decision. Nevertheless, the FDA has become more proactive in investigating the potential of Bayesian approaches in recent years. As highlighted at a workshop co-sponsored by the FDA in 2004, Bayesian designs and analyses have made a significant contribution to recent approvals of medical devices, encouraged by the fact that there is often a great deal of potentially valuable prior information in this field.

But so far, although there has been some intuitive, informal use by the FDA of prior information in regulatory decision making on investigational drugs, such as the approval of later members of the angiotensinconverting enzyme inhibitors for the treatment of heart failure, explicit Bayesian approaches to clinical trials have been lacking. Importantly, these approaches are particularly suited for therapeutic areas in which early indicators of therapeutic benefit are available. Given the rapid growth in discovery of potential biomarkers, cancer is one field in which such indicators look set to burgeon in the coming years. So, could making the most of this information through a greater application of Bayesian approaches be part of the answer to the accelerated approvals conundrum? Undoubtedly, care will be needed to preserve high scientific standards, but failure to thoroughly investigate the potential of novel clinical strategies such as Bayesian approaches could mean that 2005 will not be the nadir for new drug approvals.

1. Frantz, S. Nature Rev. Drug Discov. 4, 94-95 (2005). 